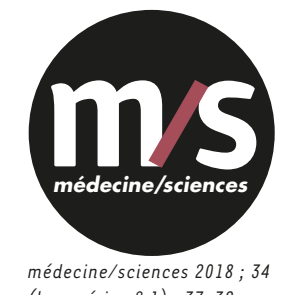

(hors série $n^{\circ} 1$ ) : 37-39

\title{
État des lieux des connaissances et des outils en génomique
}

Gaël Nicolas

> L'histoire de la génomique est relativement récente et a connu une révolution avec la complétion du séquençage du génome humain, en 2004. Il a fallu plus de dix ans pour séquencer les premiers génomes de quelques humains, impliquant un effort international majeur, des ressources financières majeures. Nous sommes ainsi entrés dans l'ère de la génomique. Nous avons 3 milliards de paires de bases qui constituent notre génome. Parmi elles, seulement 1,5\% constitue l'exome qui code directement pour la fabrication des protéines. Depuis 2009, les technologies de séquençage à haut débit ou séquençage de nouvelle génération nous donnent accès au génome/exome dans un temps relativement court.

\section{Les technologies de séquençage}

La technologie de première génération est le séquençage Sanger. Elle est extrêmement efficiente, mais des limites en termes de débit et de parallélisation se posent.

Le séquençage de nouvelle génération ajoute deux éléments. II est fait en parallèle grâce à des amorçages universels. Il est par ailleurs fait à haut débit grâce à une nouvelle technologie d'incorporation des nucléotides pour la lecture de base. On peut donc séquencer le génome entier d'un individu sur une seule cellule de détection. On peut même, dans le même test, analyser le génome de plusieurs individus en ajoutant des étiquettes moléculaires pour chaque patient.

Passer à des milliards de paires de base de données séquencées nécessite des ressources informatiques importantes, d'autant que chaque base doit être lue plusieurs fois. Les données informatiques générées sont massives, avec la nécessité de serveurs de calculs puissants et de baies de stockage importantes. Tout cela requiert un travail permanent avec les bio-informaticiens et les informaticiens. Les coûts du séquençage du génome se réduisent rapidement, mais les coûts informatiques associés restent significatifs.

Le séquençage de troisième génération, ou séquençage de longues molécules, favorise la détection des variations structurales et un phasage des variations génétiques. Cette technologie arrive dans les laboratoires de recherche depuis peu.

\section{Les grands concepts}

Ces nouvelles technologies nous permettent d'identifier de nouveaux gènes de maladies mendéliennes et de mettre en évidence l'incroyable diversité interindividuelle du génome.

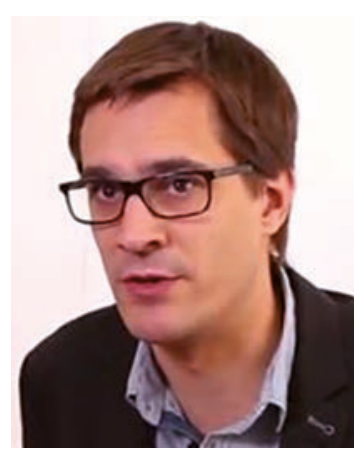

Laboratoire de génétique moléculaire, Service de génétique, Faculté de médecine et de pharmacie de Rouen, 22, boulevard Gambetta, 76183 Rouen Cedex, France. gael.nicolas@chu-rouen.fr

Concernant l'identification des bases moléculaires des maladies mendéliennes, il s'agit de séquencer les exomes ou les génomes de différentes familles et de les comparer. Il peut s'agir également du séquençage de plusieurs individus à l'intérieur d'une même famille, pour identifier directement la variation causale, alors que l'on ne connaît pas le gène causal initialement. Aujourd'hui, plus de 800 gènes pouvant causer une déficience intellectuelle, qui est une des présentations cliniques les plus hétérogènes sur le plan génétique, ont été identifiés.

\section{La découverte du polymorphisme du génome humain et de sa mutabilité}

Au début des années 2010, on bénéficiait de quelques bases de données qui permettaient d'accéder à près de 4000 génomes humains, seulement. Les nouvelles bases de données qui existent aujourd'hui contiennent plus de 130000 individus qui ont été séquencés et nous ont appris énormément. À chaque fois que l'on séquence un nouvel individu, on identifie de nouvelles variations génétiques. La nature mute en permanence, et les variations génétiques se transmettent ensuite ou non, en fonction de leur tolérance.

Les nouvelles bases de données ont également permis d'identifier la tolérance à la perte de fonction. Nous sommes tous porteurs d'une dizaine, voire d'une centaine de variations qui cassent la lecture de nos gènes et les rendent illisibles. Une mutation génétique entraînant une perte de fonction peut s'avérer délétère, non viable ou responsable d'une maladie génétique, mais peut également n'avoir aucune conséquence sur la santé. 
La mutabilité du génome a également été identifiée par le séquençage de trios. Quand on séquence le génome ou l'exome des trois individus, à savoir les parents et l'enfant, on identifie 4 millions de variations dans le génome de chaque individu, dont 20000 sont dans les exomes. Si l'on soustrait de l'enfant les variations génétiques héritées des parents, on constate les mutations nouvelles (de novo). Parmi elles, 1,5 en moyenne touche un exon et affecte donc potentiellement la structure d'une protéine (Figure 1). On sait également que plus on vieillit, plus on risque de transmettre une mutation de novo à sa descendance, ce d'autant plus que l'on est un homme. Cela est lié au nombre plus important de réplications des cellules de la lignée germinale masculine.

La mutabilité du génome cause parfois des maladies génétiques, mais il faut garder à l'esprit qu'elle est aussi nécessaire à l'adaptabilité de l'espèce. Notre génome mute et nous permet, en fonction de la pression de sélection, de conserver ou non certaines variations génétiques. En particulier, la démographie a explosé depuis 10000 ans. Cette explosion est responsable du fait que la plupart des variations génétiques sont rares, survenues récemment. Ces variations n'ont pas eu le temps de se transmettre et de diffuser dans la population.

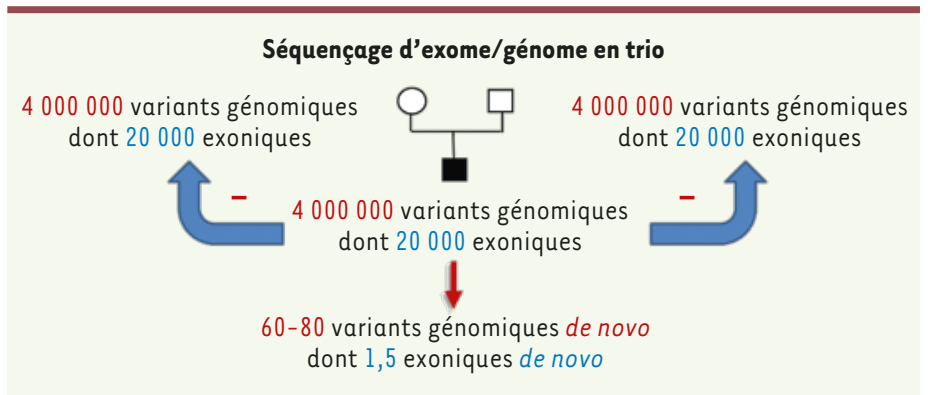

Figure 1. Variations de l'exome.

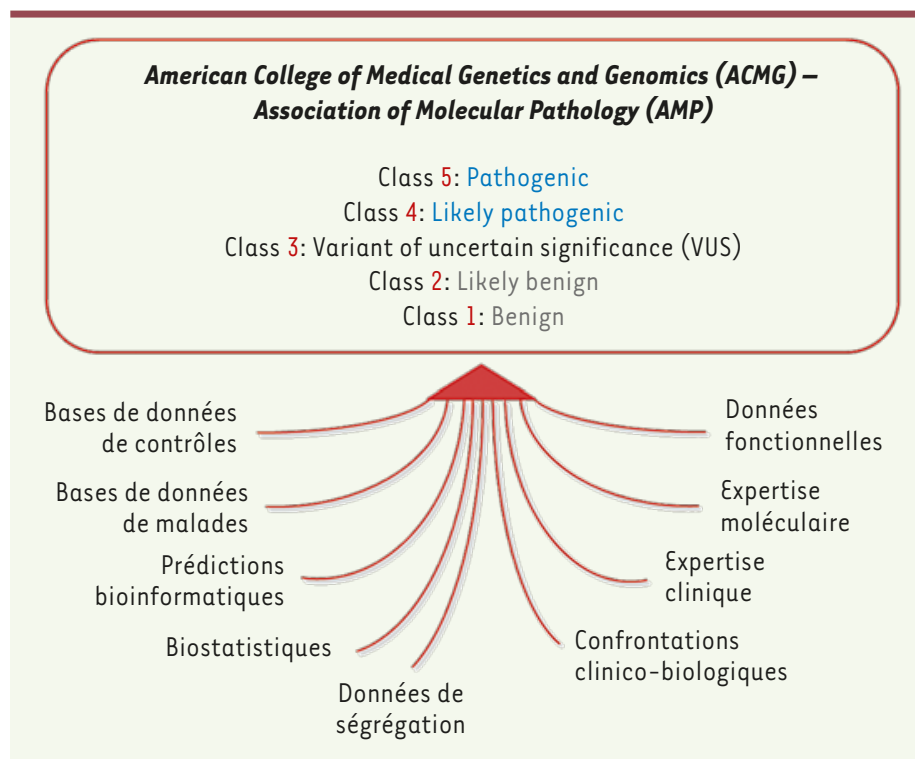

Figure 2. Recommandations de l'ACMG.
Nous savons maintenant que les mutations peuvent apparaître après la fertilisation de l'œuf et à tout stade de la vie. La technologie a donc dû s'adapter pour les détecter. Environ $7 \%$ des mutations que l'on croyait de novo germinales sont survenues en post-zygotique (post-fécondation) et ne concernent pas $100 \%$ des cellules. Le séquençage de cellules uniques a, par exemple, montré 1500 variations somatiques par neurone, alors que le neurone est une cellule post-zygotique, qui ne se divise pas. Ceci est lié à l'activité de nos neurones, qui accumulent des variations génétiques en travaillant. A contrario, les cellules de la peau et les cellules sanguines accumulent beaucoup de variations somatiques, parce qu'elles se répliquent.

\section{L'interprétation des variations génétiques et les stratégies diagnostiques}

L'exome, qui représente $1,5 \%$ du génome compte 20000 variations en moyenne par individu, dont 1000 sont considérées comme rares. Parmi celles-ci, 1,5 en moyenne est survenue de novo. C'est parmi les variations rares que l'on cherche celles qui peuvent être responsables d'une maladie génétique. Le défi n'est pas de les détecter, mais de les interpréter et de les classer.

Des recommandations américaines proposent de classer toutes les variations du génome en cinq classes: bénin, probablement bénin, de signification inconnue, probablement pathogène ou pathogène. II faut alors regrouper un ensemble d'arguments et faire preuve d'une grande expertise pour interpréter ces variants, en apportant une précision au niveau du phénotype du patient, une expertise moléculaire, bioinformatique, biostatistique, des données fonctionnelles et de la littérature (Figure 2).

Différentes stratégies peuvent être appliquées. De plus en plus, on va vers l'exome, voire le génome entier pour séquencer l'ensemble du génome et s'intéresser ensuite à un certain nombre de gènes en particulier, ce qui s'appelle des panels de gènes in silico.

Les avantages de l'exome et du génome sont la couverture, le nombre d'exons séquencés au total. Le génome est considéré comme le meilleur outil pour séquencer l'exome. Le génome est également meilleur pour détecter des variants de structure et des variations du nombre de copies (délétions, duplications).

Le séquençage de panels de gènes permet de séquencer de manière plus profonde et de détecter des variations somatiques (post-zygotiques). Lorsqu'il apparaît que le phénotype peut être lié à une mutation somatique dans un gène particulier associé un phénotype spécifique, il est encore intéressant d'avoir recours à des panels. 
En conclusion, le séquençage à haut débit représente une révolution dans la recherche, avec l'identification de la diversité du génome humain, la dissection des bases moléculaires des maladies mendéliennes ou complexes. On assiste également à une révolution médicale, du fait de la rapidité du diagnostic, de la diminution de l'errance diagnostique et de l'accès plus rapide à une prise en charge précoce, un suivi adapté, un traitement médical personnalisé et une prise en charge adaptée des familles.

L'avenir de la génomique est le développement et l'accès au séquençage du génome pour les patients. Pour cela, il nous faut mieux comprendre les variations codantes, les variations non codantes, les interactions entre les gènes et avec l'environnement, afin de permettre une médecine de précision basée sur l'interprétation des variations génomiques individuelles. $\diamond$

State of play of knowledge and of technologies in the field of genomics

\section{LIENS D'INTÉRÊT}

L'auteur déclare n'avoir aucun lien d'intérêt concernant les données publiées dans cet article.

\section{Le plan France Médecine Génomique 2025 et les maladies rares}
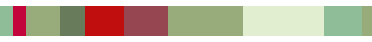

> Nous vivons une révolution en médecine grâce à la génomique, que I'on compare souvent à la révolution de la radiographie ou de l'IRM. Les outils à disposition sont, en effet, extraordinaires.

À partir de 2010, de nombreuses initiatives ont été lancées au niveau international, autour de la médecine personnalisée centrée sur les analyses génétiques, en matière de recherche et de soins. On peut notamment citer l'Initiative Genomics England, la Precision Medecine Initiative aux États-Unis et le remboursement du NGS en Estonie.

En avril 2015, le Premier ministre a chargé Yves Lévy d'étudier le problème de la médecine génomique en France et de faire des propositions. S'en est suivi un travail d'octobre 2015 à mars 2016, avec des contributions inclusives de nombreux professionnels, de beaucoup d'administrations, d'institutions, d'agences, d'industriels, d'associations de patients. Plus de 160 personnalités ont été mobilisées autour de la préparation du plan France Médecine Génomique.

Cette préparation a comporté une analyse de la situation française par rapport à la situation internationale, l'innovation et les enjeux industriels, un état des lieux en France et des propositions portant sur les infrastructures et les organisations.

Des propositions ont été formulées pour permettre à tous les patients de bénéficier de l'apport de la génomique dans le diagnostic, pour le pronostic et les soins de leur maladie, qui constituent le plan France Médecine génomique, remis en juin 2016 au Premier ministre, qui au-delà des changements gouvernementaux soutient concrètement le plan. Se sont alors mis en place les premiers axes et travaux du plan.
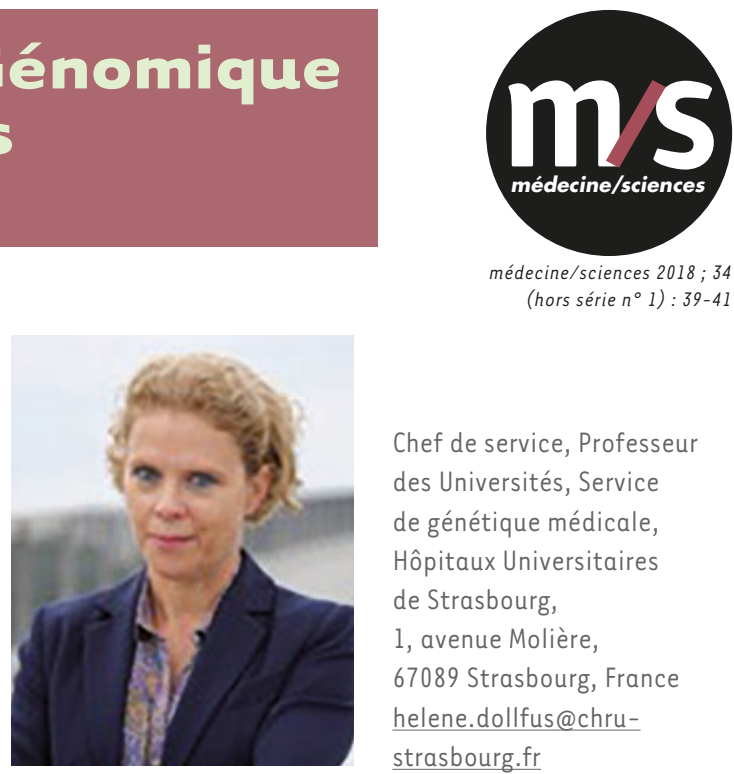

(hors série $n^{\circ}$ 1) : 39-41

Chef de service, Professeur des Universités, Service de génétique médicale, Hôpitaux Universitaires de Strasbourg, 1 , avenue Molière, 67089 Strasbourg, France helene.dollfus@chrustrasbourg.fr

\section{Les étapes de la mise en œuvre}

Le plan vise à intégrer le séquençage du génome en routine dans la pratique clinique, et à développer une filière nationale de médecine génomique en partenariat avec la recherche et les industriels (Figure 1). La France veut en effet redevenir leader dans ce domaine.

Le plan est organisé autour de trois objectifs et 14 mesures. II s'adresse à toutes les maladies. Certains objectifs sont génériques et seront utilisables dans le domaine du cancer comme celui des maladies rares.

Tout d'abord, il a été décidé, pour structurer le séquençage haut débit, de mettre en place des plateformes au niveau du territoire dans les prochaines années. Ces plateformes sont supposées couvrir l'ensemble des pathologies susceptibles d'avoir besoin de séquençage haut débit. En décembre 2016, un appel à projets a été lancé à l'attention des plateformes. Deux plateformes de séquençage ont été sélectionnées: SEDOiA 